\title{
Optimal sequences of trials for balancing practice and repetition effects
}

\author{
HAN-SUK SOHN, DENNIS L. BRICKER, and J. RICHARD SIMON \\ University of Iowa, Iowa City, Iowa \\ and \\ YI-CHIH HSIEH \\ National Yunlin Polytechnic Institute, Yunlin, Taiwan
}

\begin{abstract}
This paper describes procedures for generating trial sequences to balance out practice effects and intertrial repetition effects in experiments consisting of repeated trials. In the sequences presented, each stimulus appears an equal number of times, is preceded equally often by itself and by each other stimulus, and is distributed in a "balanced" manner throughout the block of trials. Two criteria for balance are employed. One criterion aims to equalize the average position of each stimulus in the sequence. The second criterion maintains, as much as possible, a uniform interval between appearances of each stimulus in the sequence. For each criterion, optimal or near-optimal sequences are presented for experiments involving from three to nine different stimulus conditions. Suggestions are included for extending (e.g., doubling or tripling) the length of the sequences.
\end{abstract}

A typical reaction time (RT) experiment consists of a series of trials. On each trial, the subject selects and executes a response depending on the identity of the stimulus presented. Typically, each stimulus is presented a number of times in some predetermined or randomly generated sequence. The experimenter computes the mean or median RTs and error rates for each of the different stimulus conditions to examine the effects of the experimental treatment.

When subjects perform repeated trials on a speeded choice task, their RT on a particular trial may be affected by several factors other than the specific stimulus presented. For example, performance may be affected by practice effects that emerge within a block or over several blocks of trials (Pashler \& Baylis, 1991b; Woodworth, 1938). There may also be an intertrial repetition effect, in which performance on a given trial is affected by the nature of the preceding trial (see, e.g., Bertelson, 1961; Pashler \& Baylis, 1991a; Smith, 1968; Williams, 1966). In addition, RT may be affected by the probability of stimulus occurrence (Hinrichs \& Craft, 1971).

In an attempt to control these possible sources of confounding within a block of trials, experimenters generally present each stimulus equally often in a random sequence. Emerson and Tobias (1995) recently presented a computer program for randomly generating sequences of

H.-S.S. and D.L.B. are in the Department of Industrial Engineering, University of Iowa; J.R.S. is in the Departments of Psychology and Industrial Engineering, University of Iowa; and Y.-C.H. is in the Department of Industrial Engineering, National Yunlin Polytechnic Institute, Yunlin, Taiwan. Correspondence should be addressed to D. L. Bricker, Department of Industrial Engineering, University of lowa, Iowa City, IA 52242 (e-mail: dennis-bricker@uiowa.edu). trials so that each stimulus condition (1) appears an equal number of times and (2) is preceded equally often by itself and by each of the other stimuli (Emerson \& Tobias, 1995). The number of possible sequences exhibiting these properties is, in general, quite large; the algorithm of Emerson and Tobias produces sequences with equal-probability sampling.

An alternative control strategy would be to employ sequences of trials that are optimal in the sense that each stimulus condition, in addition to satisfying properties (1) and (2) above, is as evenly distributed as possible throughout the block of trials. This paper describes criteria for optimality of these sequences and presents optimal sequences for experiments involving from three to nine treatment conditions, with each preceded exactly once by each other condition, including itself. We suggest that experimenters might employ these sequences to balance out irrelevant effects in experiments involving repeated trials. The sequences presented here should also be useful to experimenters who wish to systematically investigate the intertrial repetition effect.

\section{MATHEMATICAL STATEMENT OF THE PROBLEM}

The subsequence $\{i, j\}$ must appear exactly once in the complete sequence. Denote by $O_{i j}$ the position of this pair in the sequence-that is, $O_{i j}=k$ if $i$ appears in the $k$ th position, followed by $j$ in the $(k+1)$ th position.

Denote by $S_{i}$ the sum of the positions of $i$ in the sequence-that is,

$$
S_{i}=\sum_{j=1}^{N} O_{i j} .
$$


Consider the sum of these sums,

$$
\sum_{i=1}^{N} S_{i}=\sum_{i=1}^{N} \sum_{j=1}^{N} O_{i j}
$$

Note that the set of all the numbers $O_{i j}$ is some permutation of $1,2, \ldots, N^{2}$, and so their sum is identical to that of a simple arithmetic sequence and is easily computed:

$$
\sum_{i=1}^{N} S_{i}=1+2+\cdots+N^{2}=\frac{N^{2}\left(N^{2}+1\right)}{2} .
$$

The average of the values of $S_{i}, i=1,2, \ldots, N$, is therefore

$$
\tau=\frac{N^{2}\left(N^{2}+1\right)}{2} /_{N}=\frac{N\left(N^{2}+1\right)}{2} .
$$

We may consider $i$ to be "balanced" in the sequence if the sum of its positions, $S_{i}$, is equal to this "target" value, $\tau$, or, equivalently, if the deviation from the target,

$$
\delta_{i}=S_{i}-\tau,
$$

is zero. Unfortunately, it is generally impossible to obtain a sequence satisfying our restrictions that attains a perfect balance of all of the integers $1,2, \ldots, N$. It is necessary to introduce some additional concepts before discussing the criterion, which we will use to select the "best" sequence.

For each sequence $O$, define its position cardinality vector $\boldsymbol{\kappa}(O)=\left(\kappa_{0}, \kappa_{1}, \kappa_{2}, \ldots\right)$ by

$$
\boldsymbol{\kappa}_{n}=m \Leftrightarrow \operatorname{Card}\left\{i:\left|\delta_{i}\right|=n\right\}=m,
$$

where $\operatorname{Card}(X)$ is the cardinality of the set $X$-that is, the number of elements in the set.

This cardinality vector is a good indicator of the balance of distribution of the integers in the sequence, where $\boldsymbol{\kappa}=(N, 0,0,0, \ldots)$ would indicate a perfect balancethat is, a sequence for which each position sum $S_{i}(i=1$, $\ldots, n$ ) has zero deviation $\delta_{i}$ from the target $\tau$.

A vector $\boldsymbol{\alpha}$ is said to be lexicographically positive, denoted

$$
\boldsymbol{\alpha}>0,
$$

if its first nonzero element is positive, and vector $\boldsymbol{\alpha}$ is said to be lexicographically greater than vector $\boldsymbol{\beta}$, denoted

$$
\boldsymbol{\alpha}>\boldsymbol{\beta},
$$

if the difference $\boldsymbol{\alpha}-\boldsymbol{\beta}$ is lexicographically positive. (We define "lexicographically less than" accordingly; that is,

$$
\left.\boldsymbol{\alpha}<\boldsymbol{\beta} \Leftrightarrow \boldsymbol{\beta}>_{\mathrm{L}} \boldsymbol{\alpha}\right) .
$$

We will also require the reversal operator $\rho$ for a vector, which we define in the obvious way-that is, for any vector $\boldsymbol{\alpha}=\left(\alpha_{0}, \alpha_{1}, \alpha_{2}, \ldots, \alpha_{n}\right)$, the reverse of the vector is $\rho(\boldsymbol{\alpha})=\left(\alpha_{n}, \alpha_{n-1}, \ldots, \alpha_{1}, \alpha_{0}\right)$. The criterion that we propose for measuring the balance of the sequence of integers is the position cardinality vector introduced above. Specifically, we wish to lexicographically minimize the reverse of the cardinality vector,

$$
\underset{O}{\operatorname{Lexmin}} \rho[\boldsymbol{\kappa}(O)]
$$

that is, to find a sequence $O^{*}$ with cardinality vector $\boldsymbol{\kappa}^{*}=\boldsymbol{\kappa}\left(O^{*}\right)$ such that, if $O^{\prime}$ is any other feasible sequence with cardinality vector $\boldsymbol{\kappa}^{\prime}=\boldsymbol{\kappa}\left(O^{\prime}\right)$, then

$$
\rho\left(\boldsymbol{\kappa}^{*}\right)<\rho\left(\boldsymbol{\kappa}^{\prime}\right) .
$$

Note that applying this criterion minimizes not only

$$
\max _{i}\left|\delta_{i}\right|
$$

the maximum of the absolute deviations for the integers, but also the number of integers exhibiting this maximum absolute deviation.

\section{EXAMPLE}

Consider the case $N=3$. The sequence (3-) 1-1-2$3-3-2-2-1-3$ is a feasible sequence; that is, each of the integers 1,2 , and 3 appears three times and is preceded once by each of the others and itself. (In general, we require that the subject be presented the preamble shown within parentheses, identical to the final integer of the sequence, namely 3 in this instance, but the observation for that preliminary trial is to be discarded.) The matrix $O$ for this sequence is

$$
O=\left[\begin{array}{lll}
1 & 2 & 8 \\
7 & 6 & 3 \\
9 & 5 & 4
\end{array}\right] .
$$

That is, the pair 1-1 appears first in the sequence 1-1-23-3-2-2-1-3, followed by the pair 1-2, while 1-3 appears in the eighth position, so that 1 appears in the first, second, and eighth positions, 2 appears in the seventh, sixth, and third positions, and so on. The row sums of the matrix $O$ therefore are $S_{1}=11, S_{2}=16$, and $S_{3}=18$, while the target $\tau$, based on Equation 4, is 15. (This implies that 1 and 3 are less balanced than 2 ; i.e., $S_{1}<\tau$ implies that the appearances of 1 tend to be concentrated early in the sequence, while $S_{3}>\tau$ implies that the appearances of 3 tend to be concentrated late in the sequence.) Thus, the deviations from the target are $\delta_{1}=-4, \delta_{2}=$ +1 , and $\delta_{3}=+3$, and the cardinality vector is $\kappa=(0,1$, $0,1,1,0,0, \ldots)$; that is, there are no deviations of magnitude zero, one of magnitude 1 , none of magnitude 2 , and so on.

Compare the sequence above with the (optimal) sequence (1-) 1-2-3-1-3-3-2-2-1, for which the matrix $O$ is

$$
O=\left[\begin{array}{lll}
9 & 1 & 4 \\
8 & 7 & 2 \\
3 & 6 & 5
\end{array}\right]
$$


and $S_{1}=S_{3}=14$, and $S_{2}=17$. The deviations from $\tau$ are now $\delta_{1}=\delta_{3}=-1$ and $\delta_{2}=+2$, and the cardinality vector is $\boldsymbol{\kappa}^{*}=(0,2,1,0,0, \ldots)$.

Comparing the latter cardinality vector with the former, we see that

$$
\rho\left(\boldsymbol{\kappa}^{*}\right) \underset{\mathrm{L}}{<} \rho(\boldsymbol{\kappa})
$$

since the reversed difference

$$
\rho\left(\kappa-\kappa^{*}\right)=(\ldots, 0,0,1,1,-1,-1,0)>0
$$

that is, the cardinality of the largest deviation for the first sequence $\left(\kappa_{4}=1\right)$ is greater than that of the corresponding deviation of the second sequence, namely zero, so that the first nonzero element of $\rho\left(\kappa-\kappa^{*}\right)$ is positive. For this reason, we will consider the latter sequence to be better balanced and hence superior to the former.

\section{OPTIMALLY BALANCED SEQUENCES}

The program is written in the APL*PLUS II language and is run on the HP-UX 715/75 workstation. Although the number of distinct sequences of the integers 1 through $N$, each repeated $N$ times, is

$$
\frac{\left(N^{2}\right) !}{N(N !)}
$$

the number of such sequences that are feasible is "only" $(N !)^{N}$. It is easy to see that the complexity of this problem precludes a complete enumeration of the feasible sequences and limits the size of the problems that can be solved within a practicable amount of computation time. For example, the number of feasible sequences when $N=3$ is only 216 , but for $N=4,5$, and 6 , this number grows rapidly to $331776,2.488 \times 10^{10}$, and $1.39 \times 10^{17}$, respectively. The program therefore utilizes an implicit enumeration algorithm to severely restrict the number of sequences that require evaluation. Even so, for $N>6$, the time required for the search was excessive, and the search was terminated at $86,400 \mathrm{sec}$, so that the optimality of all given sequences cannot be guaranteed. Table 1 presents,

Table 1

Sequences (A) Exhibiting a Balance of Integers

\begin{tabular}{llllllll}
\hline$N$ & \multicolumn{6}{c}{ Sequence } \\
\hline $3^{*}$ & 12313 & 3221 \\
$4^{*}$ & 12344 & 13324 & 22114 & 3 & & \\
$5^{*}$ & 12345 & 43352 & 21551 & 14424 & 13253 & \\
$6^{*}$ & 12345 & 66554 & 33221 & 63146 & 41152 & 62442 \\
& 51353 & 6 & & & & \\
7 & 12345 & 67766 & 55443 & 32211 & 31747 & 36272 \\
& 51575 & 24616 & 41426 & 3537 & & \\
8 & 12345 & 67887 & 76655 & 44332 & 21131 & 42418 \\
& 28385 & 81627 & 57374 & 68635 & 26172 & 51536 \\
& 4847 & & & & & & \\
9 & 12345 & 67899 & 88776 & 65544 & 33224 & 21131 \\
& 91595 & 39352 & 92646 & 27471 & 73868 & 18485 \\
& 79697 & 58283 & 61494 & 16372 & 5 & & \\
\hline
\end{tabular}

Note $-N^{*}$ indicates that optimality of the sequence is guaranteed.

\begin{tabular}{|c|c|c|c|c|c|c|c|c|c|}
\hline \multirow[b]{2}{*}{$N$} & \multicolumn{9}{|c|}{ Deviations $\delta_{i}=S_{i}-\tau$} \\
\hline & 1 & 2 & 3 & 4 & 5 & 6 & 7 & 8 & 9 \\
\hline 3 & -1 & 2 & -1 & & & & & & \\
\hline 4 & 0 & 0 & 0 & 0 & & & & & \\
\hline 5 & 0 & 0 & 0 & 0 & 0 & & & & \\
\hline 6 & 0 & 0 & 0 & 0 & 0 & 0 & & & \\
\hline 7 & 0 & 0 & 0 & 1 & -1 & 0 & 0 & & \\
\hline 8 & -13 & -14 & -12 & 0 & 14 & 15 & 12 & -2 & \\
\hline 9 & 3 & -18 & -20 & -3 & -15 & 21 & 17 & 22 & -7 \\
\hline
\end{tabular}

Table 2

Deviations $\delta$ From Target $\tau$ of the Sequences (A) in Table 1

for values of $N$ ranging from 3 to 9 , the best sequences found. $N^{*}$ denotes that the optimality of the sequence is guaranteed for $N=3,4,5$, and 6 . To distinguish these sequences from other sequences to be introduced later, we shall refer to them as sequences of Type A.

Table 2 indicates the balance of those sequences; namely, it reports the deviations $\delta$ of the the average position of each condition from the target $\tau$.

\section{EXTENDING THE OPTIMAL SEQUENCES}

For small values of $N$, the experimenter may wish to construct a longer sequence by repeating conditions more than $N$ times. For example, when $N$ is 3 , the length of the sequence reported in Table 1 is only $N^{2}=9$. How may the experimenter double or triple the length of the sequence, for example, and still obtain a sequence with a quality as good as or better than the given sequence? With this goal in mind, we state the following three properties.

Property 1. Let $O^{\prime}=\rho(O)$ denote the sequence obtained by reversing sequence $O$, with row sums $S_{i}^{\prime}$ and deviations from the target $\delta_{i}^{\prime}$. Then

$$
\begin{aligned}
S_{i}^{\prime} & =\sum_{j=1}^{N} O_{i j}^{\prime}=\sum_{j=1}^{N}\left(N^{2}+1-O_{i j}\right) \\
& =N\left(N^{2}+1\right)-\sum_{j=1}^{N} O_{i j}=2 \tau-S_{i} \\
\delta_{i}^{\prime} & =S_{i}^{\prime}-\tau=\left(2 \tau-S_{i}\right)-\tau=-\delta_{i} .
\end{aligned}
$$

Property 2. Let $O^{\prime \prime}=\operatorname{cat}\left(O, O^{\prime}\right)$ denote the sequence obtained by concatenating sequence $O^{\prime}$ to the end of sequence $O$, with row sums $S_{i}^{\prime \prime}$ and deviations from the target $\delta_{i}^{\prime \prime}$. The target for the row sums is now

$$
\tau^{\prime \prime}=\frac{1}{N} \sum_{k=1}^{2 N^{2}} k=\frac{1}{N} \frac{2 N^{2}\left(2 N^{2}+1\right)}{2}=2 N^{3}+N=N^{3}+2 \tau
$$

and

$$
\begin{aligned}
& S_{i}^{\prime \prime}=\sum_{j=1}^{N}\left[O_{i j}+\left(N^{2}+O_{i j}^{\prime}\right)\right]=S_{i}+S_{i}^{\prime}+N^{3} \\
& \delta_{i}^{\prime \prime}=S_{i}^{\prime \prime}-\tau^{\prime \prime}=\left(S_{i}+S_{i}^{\prime}+N^{3}\right)-\left(2 \tau+N^{3}\right)=\delta_{i}+\delta_{i}^{\prime} .
\end{aligned}
$$

Property 3. Let $\pi$ denote a permutation of the set of integers $\{1,2, \ldots, N\}$, which provides us a one-to-one mapping of the set onto itself; that is, $\pi=\left\{\pi_{1}, \pi_{2}, \ldots\right.$, 
$\left.\pi_{N}\right\}$. Denote by $O^{\prime}=\pi(O)$ the sequence obtained by this mapping; that is, $O_{\pi i, \pi j}^{\prime}=O_{i j}$. Then

$$
S_{\pi_{i}}^{\prime}=S_{i} \text { and } \delta_{\pi_{i}}^{\prime}=\delta_{i} \text {. }
$$

By means of these properties, we will be able to extend (e.g., double or triple) the length of a sequence without degrading the quality of the sequence and usually improving the quality.

\section{EXAMPLE}

Consider again the case $N=3$ and the sequence (1-) $1-2-3-1-3-3-2-2-1$ with $\delta_{1}=\delta_{3}=-1$ and $\delta_{2}=+2$. (Recall that the 1 in parentheses indicates that the observation for that initial trial is to be discarded.) A valid sequence may always be obtained from any sequence by repeating it, obtaining, in this case, (1-) 1-2-3-1-3-3$2-2-1-1-2-3-1-3-3-2-2-1$. (Note that the final 1 in the sequence serves as the preamble to the repetition of the sequence.) Thus we have doubled the length of the sequence, but according to Property 2 we have also doubled the deviations from the target, obtaining $\delta_{1}^{\prime}=\delta_{3}^{\prime}=$ -2 and $\delta_{2}^{\prime}=+4$. For $N=4,5$, and 6 , however, the optimal sequences given in Table 1 are perfectly balanced, so the repetitions of the optimal sequence in these cases are also optimal.

When the initial condition and final condition of a sequence are identical to that sequence, we can always concatenate its reverse, obtaining for the case $N=3$ in Table 1, for example, (1-) 1-2-3-1-3-3-2-2-1-1-2-2-3$3-1-3-2-1$. Since, by Property 1 , reversing a sequence also reverses the signs of the deviations $\delta$ from the target $\tau$, and by Property 2 , the deviations $\delta^{\prime \prime}$ resulting from concatenation are the sums of the deviations $\delta$ and $\delta^{\prime}$ of the concatenated sequences, the resulting sequence, $O^{\prime \prime}=$ $\operatorname{cat}\left(O, O^{\prime}\right)$, always has $\delta_{i}^{\prime \prime}=0, i=1,2, \ldots, N$, that is, it is perfectly balanced, even if the original sequence was not! Note that any reversible sequence (not necessarily perfectly balanced) could be used for this doubling operation to yield a perfectly balanced sequence of length $2 N^{2}$; such reversible sequences will appear later in Tables 3 and 5 .

Once such a doubled sequence has been obtained, it can be repeated as often as desired, so that one can obtain perfectly balanced sequences of any length that is an even multiple of $N^{2}$. If, on the other hand, one wishes a sequence with length an odd multiple of $N^{2}$, the sequence cat $[O, \rho(O), \ldots, O, \rho(O), O]$ has a deviation vector equal to the original $\delta$. In this case, then, the extended sequence is no better balanced than the original, but neither is it worse.

One may wish to preclude the negative effects of possible sequence learning by the subject in the case of extended sequences of length greater than $2 N^{2}$. Instead of constructing an extended sequence by repeated concatenations of $O$ and its reverse $\rho(O)$, equally well-balanced sequences might be obtained by a combination of a permutation of the original sequence $O$ and its reverse. Con- sider the case $N=3$, for example, in which the perfectly balanced doubled sequence is (1-) $1-2-3-1-3-3-2-2-$ $1-1-2-2-3-3-1-3-2-1$. If we perform any permutation of the stimuli excluding 1 , we obtain another perfectly balanced sequence. For example, let $\pi=(1,3,2)$, so that $\pi$ applied to cat $[O, \rho(O)]$ yields the sequence (1-) 1-2-2-3-3 $-2-2-1-1-3-1-2-3-3-2-1-3-1$, which, when concatenated to cat $[O, \rho(O)]$, yields a perfectly balanced sequence of length $4 N^{2}$. By other proper choices of permutations, perfectly balanced sequences with length equal to any even multiple of $N^{2}$ can be obtained. (A sequence that has length an odd multiple of $N^{2}$ can be obtained by concatenating the original $O$ to a perfectly balanced sequence whose construction was just described.)

\section{AN ALTERNATIVE CRITERION}

Although the previous criterion may be appropriate for the planning of most experiments, in some cases there may be a need to maintain, as much as possible, a uniform interval between appearances of each number in the sequence. The criterion above in Equation 7 makes no attempt to do this and may yield sequences in which, for example, a number may appear in clusters at both ends of the sequence, thereby achieving a balance relative to the center of the sequence.

Denote by $G_{i j}$ the size of gap $j$ for each integer in the sequence - that is, the interval between the $j$ th presentation of $i$ and the $(j+1)$ th presentation of that same $i, j=1,2$, $\cdots, n-1$, where the first integer $i$ in the sequence is considered to be preceded by the last. Then we would like to increase the uniformity of $G_{i j}$, or equivalently, to reduce the variability of $G_{i j}$ about its mean, which is always $N$.

For each sequence $O$, define the gap cardinality vector $\gamma(O)=\left(\gamma_{0}, \gamma_{1}, \gamma_{2}, \cdots\right)$ by

$$
\begin{aligned}
\gamma_{k} & =m \Leftrightarrow \operatorname{Card}\left\{(i, j):\left|G_{i j}-N\right|\right. \\
& =k, j=1, \cdots n-1\}=m ;
\end{aligned}
$$

that is, $\gamma_{k}$ is the number of deviations from the mean $N$ of gaps or intervals between the repetitions of each $i$ that is of magnitude $k, k \geq 0$. Hence, we propose this gap cardinality vector $\gamma(O)$ as a measure of the nonuniformity of the sequence of integers, whose reverse may accordingly be lexicographically minimized-that is,

$$
\underset{O}{\operatorname{Lexmin}} \rho[\gamma(\mathrm{O})]
$$

Optimizing this criterion will not only minimize max $\left\{k: \gamma_{k}>0\right\}$, the maximum deviation of gaps from the desired value $N$, but will further minimize the number of such gaps.

\section{EXAMPLE}

Let us consider again the first feasible sequence presented above for $N=3$, namely (3-) $1-1-2-3-3-2-2-$ $1-3$. The values of $G_{i f}$ and $\left|G_{i f}-N\right|$ in this sequence are 


$$
\left[\begin{array}{ll}
1 & 6 \\
3 & 1 \\
1 & 4
\end{array}\right] \text { and }\left[\begin{array}{ll}
2 & 3 \\
0 & 2 \\
2 & 1
\end{array}\right],
$$

respectively. (For example, $G_{32}$, i.e., the gap between the second and third presentations of the number 3 , which, ignoring the preamble, is presented in Positions 4, 5, and 9 is $9-5=4$, which differs from the mean [namely, $N=3$ ] by $\left|G_{32}=N\right|=|4-3|=1$.) The cardinality vector of $\left|G_{i f}-N\right|$ is $\gamma=\left\{\gamma_{0}, \gamma_{1}, \gamma_{2}, \gamma_{3}, \ldots\right\}=\{1,1,3,1,0$, ... $\}$ since 0 and 1 appear once each, 2 appears three times, and 3 appears once.

Compare the above sequence with the optimal sequence for this criterion, namely $(1-) 1-2-3-2-2-1-3-3-1$. The values of the gaps $G_{i j}^{*}$ and the deviations $\left|G_{i j}^{*}-N\right|$ in this sequence are

$$
\left[\begin{array}{ll}
3 & 5 \\
1 & 2 \\
1 & 4
\end{array}\right] \text { and }\left[\begin{array}{ll}
0 & 2 \\
2 & 1 \\
2 & 1
\end{array}\right] \text {, }
$$

respectively, so that the cardinality vector of $\left|G_{i j}^{*}-N\right|$ is $\boldsymbol{\gamma}^{*}=\left\{\gamma_{0}, \gamma_{1}, \gamma_{2}, \gamma_{3}, \ldots\right\}=\{1,1,4,0,0, \ldots\}$. The reverse of the gap cardinality vector of the former-namely, $\rho(\gamma)$, is $(1,1,3,1,0, \ldots)$-while that of the latter is $\rho\left(\gamma^{*}\right)=$ $(1,1,4,0,0, \ldots)$. Since the leftmost nonzero element of the difference $\rho(\gamma)-\rho\left(\gamma^{*}\right)=(\ldots 0,+1,-1,0,0)$ is positive, $\rho(\gamma)$ is lexicographically greater than $\rho\left(\gamma^{*}\right)$ and therefore, based upon criterion (Equation 8 ), we would consider the second sequence to be better than the first sequence.

\section{UNIFORMLY DISTRIBUTED SEQUENCES}

Table 3 presents, for values of $N$ ranging from 3 to 9 , sequences selected based upon the second criterion (Equation 8). These will be referred to as Type B sequences. $N^{*}$ in Table 3 indicates that the optimality of the sequence is guaranteed. Otherwise, the search was terminated at $86,400 \mathrm{sec}$, so that, as before, the optimality of the sequence cannot be guaranteed. Table 4 indicates the quality of those sequences, namely the gap cardinality vector of each sequence.

Table 3

Sequences (B), Exhibiting Even Distribution of Integers

\begin{tabular}{llllllll}
\hline$N$ & \multicolumn{5}{c}{ Sequence } \\
\hline $3^{*}$ & 12322 & 1331 & & & \\
$4^{*}$ & 12344 & 13243 & 31422 & 1 & & \\
$5^{*}$ & 12345 & 22143 & 54251 & 32441 & 55331 & \\
6 & 12345 & 61324 & 65316 & 64263 & 51433 & 62544 \\
& 15522 & 1 & & & & & \\
7 & 12345 & 67132 & 46573 & 14263 & 51743 & 36254 \\
& 15377 & 21644 & 75522 & 7661 & & \\
8 & 12345 & 67813 & 24657 & 18362 & 54731 & 68275 \\
& 38417 & 63526 & 48514 & 33744 & 28661 & 55887 \\
& 7221 & & & & & & \\
9 & 12345 & 67891 & 32465 & 79814 & 36259 & 71842 \\
& 63519 & 47286 & 15392 & 74852 & 16958 & 22937 \\
& 76441 & 73875 & 54996 & 68833 & 1 & \\
\hline
\end{tabular}

Note $-N^{*}$ indicates that optimality of the sequences is guaranteed.
Table 4 Gap Cardinality Vectors Optimal Gap Cardinality Vectors $\gamma^{*}$

\begin{tabular}{rrrrrrrrrr}
$N$ & $\gamma_{0}$ & $\gamma_{1}$ & $\gamma_{2}$ & $\gamma_{3}$ & $\gamma_{4}$ & $\gamma_{5}$ & $\gamma_{6}$ & $\gamma_{7}$ & $\gamma_{8}$ \\
\hline 3 & 1 & 2 & 3 & & & & & & \\
4 & 4 & 2 & 3 & 3 & & & & & \\
5 & 3 & 6 & 7 & 0 & 4 & & & & \\
6 & 4 & 12 & 4 & 5 & 0 & 5 & & & \\
7 & 6 & 17 & 7 & 2 & 3 & 1 & 6 & & \\
8 & 8 & 15 & 12 & 6 & 5 & 3 & 0 & 7 & \\
9 & 15 & 18 & 14 & 3 & 6 & 2 & 2 & 4 & 8 \\
\hline
\end{tabular}

\section{EXTENDING THE UNIFORMLY DISTRIBUTED SEQUENCES}

As discussed before, longer sequences than those given in Table 3 might be required. The following properties will prove useful in extending the sequences:

Property 4. Let $O^{\prime}=\rho(O)$ denote the sequence obtained by reversing sequence $O$. Then the gap cardinality vector of the reversed sequence is identical to the original sequence-that is, $\gamma\left(O^{\prime}\right)=\gamma(O)$.

Property 5. Let $\pi$ denote a permutation of the set of integers $\{1,2, \ldots, N\}$, which provides us a one-to-one mapping of the set onto itself; that is, $\pi=\left\{\pi_{1}, \pi_{2}, \ldots\right.$, $\left.\pi_{N}\right\}$. Denote by $O^{\prime}=\pi(O)$ the sequence obtained by this mapping; that is, $O_{\pi i, \pi j}^{\prime}=O_{i j}$. Then the gap cardinality vector is unchanged by this permutation; that is, $\gamma\left(O^{\prime}\right)=\gamma(O)$.

Properties 4 and 5 might lead one to expect that concatenating the reverse or some permutation thereof might yield good extended sequences, an expectation that is shown to be unwarranted because of the fact that concatenating two sequences not only retains the gaps in each sequence, but also introduces additional gaps, namely the gap between the final appearance of a number in the first sequence and its first appearance in the next. For the purpose of generating sequences that when repeated continue to display a uniform distribution of the stimuli, we extend the definition of the gap $G_{i j}$ to include the case $j=n$ by letting $G_{i n}$ denote the interval between the $n$th appearance of integer $i$ and the $(n+1)$ th appearance when the sequence is repeated. We distinguish the new gap cardinality vector that includes a count of these additional gaps by $\gamma^{O}$ (suggesting the circularity of the gaps). Denote by $\phi^{k}(O)$ the sequence obtained by rotation of each element of the sequence $O$ (in a circular sense) $k$ positions to the left. [Thus, if $O$ represents the sequence (1-) $1-2-$ 3-2-2-1-3-3-1 from Table 3, $\phi^{\prime}(O)$ is the sequence (1-) $2-3-2-2-1-3-3-1-1$.] The new gap cardinality vector $\gamma^{o}$ has the following three useful properties:

Property 6. Let $O^{\prime}=\pi(O)$ be a permutation of sequence $O$. Then $\gamma^{O}\left(O^{\prime}\right)=\gamma^{O}(O)$; that is, the gap cardinality vector $\gamma^{O}$ is unchanged by this permutation.

Property 7. $\boldsymbol{\gamma}^{O}(O)=\boldsymbol{\gamma}^{O}\left[\phi^{k}(O)\right]$; that is, $\boldsymbol{\gamma}^{O}$ is unaffected by a rotation. 
Table 5

Circular Sequences (Type C),

Exhibiting Even Distribution of Integers

\begin{tabular}{llllllll}
\hline$N$ & \multicolumn{5}{c}{ Sequence } \\
\hline $3^{*}$ & 12231 & 3321 & & & \\
$4^{*}$ & 12344 & 13224 & 33142 & 1 & & & \\
5 & 12345 & 13522 & 44153 & 32142 & 55431 & \\
6 & 12345 & 61324 & 65143 & 66225 & 31542 & 16335 \\
& 52644 & 1 & & & & & \\
7 & 12344 & 55266 & 77315 & 46271 & 35642 & 17536 \\
& 14725 & 16332 & 24376 & 5741 & & \\
8 & 12345 & 67813 & 24657 & 18362 & 54731 & 68275 \\
& 38417 & 63528 & 87422 & 15866 & 44337 & 72614 \\
& 8551 & & & & & \\
9 & 12345 & 67891 & 32465 & 79814 & 36259 & 71842 \\
& 63519 & 47286 & 15392 & 74852 & 16937 & 58773 \\
& 82295 & 54176 & 64499 & 68833 & 1 & \\
\hline
\end{tabular}

Note- $N^{*}$ indicates that optimality of the sequences is guaranteed.

Table 6

Optimal Circular Gap Cardinality Vectors $\gamma^{o}$

\begin{tabular}{rrrrrrrrrrrrrr}
\hline$N$ & $\gamma_{0}^{O}$ & $\gamma_{1}^{O}$ & $\gamma_{2}^{O}$ & $\gamma_{3}^{O}$ & $\gamma_{4}^{O}$ & $\gamma_{5}^{O}$ & $\gamma_{6}^{O}$ & $\gamma_{7}^{O}$ & $\gamma_{8}^{O}$ & $\gamma_{9}^{O}$ & $\gamma_{10}^{O}$ & $\gamma_{11}^{O}$ & $\gamma_{12}^{O}$ \\
\hline 3 & 1 & 3 & 4 & 1 & & & & & & & & & \\
4 & 3 & 4 & 3 & 6 & & & & & & & & \\
5 & 2 & 5 & 10 & 3 & 5 & & & & & & & \\
6 & 7 & 10 & 3 & 4 & 6 & 6 & & & & & & \\
7 & 7 & 15 & 7 & 5 & 4 & 0 & 11 & & & & & \\
8 & 12 & 17 & 10 & 6 & 3 & 5 & 0 & 10 & 1 & & & & \\
9 & 17 & 17 & 18 & 4 & 4 & 2 & 2 & 1 & 10 & 1 & 2 & 3 \\
\hline
\end{tabular}

Property 8. Let $O^{\prime}=\operatorname{cat}(O, O)$ denote the sequence obtained by repeating sequence $O$. Then the gap cardinality vector of the extended sequence is $\gamma^{O}\left(O^{\prime}\right)=2 \gamma^{O}(O)$.

Table 5 displays sequences (henceforth referred to as Type C) that exhibit an even distribution of integers in this new sense, namely the cardinality vector of the gaps as determined in this circular sense (see Table 6). (Note that each sequence shown in Table 5 is reversible.) These Type $\mathrm{C}$ sequences may be extended by concatenating a sequence to itself, which yields a sequence with the same uniformity of distribution of the integers as the original sequence.

\section{DISCUSSION}

In this paper we have suggested two techniques for measuring the quality of sequences of trials according to two criteria - namely (1) the balance of trials within the sequence, so as to avoid the influence of practice effects during the block of trials, and (2) uniformity of the spacing of the trials to avoid intertrial repetition effects. The degree to which one or the other criterion deserves serious consideration in an experimental design depends upon the nature and purpose of the specific experiment, of course. For each criterion we have presented optimal or near-optimal sequences, from which by various transformations (e.g., reversal, rotations, and/or permutations, depending upon the criterion) one may obtain other sequences having equal quality that might be used as additional blocks of trials. Many experimenters may, of course, wish to give weight to both criteria. Table 7 will be of interest to the experimenter who, having selected a sequence from Tables 1 or 3 above according to his/her primary criterion, may wish to know the quality of that sequence as judged by the other criterion. When both criteria are deemed to be important, a reference to Table 7 indicates the sacrifice one makes in satisfying the secondary criterion on the basis of one's choice of a primary criterion. (Note that Table 7 reports only

$$
\max _{i}\left|\delta_{i}\right|
$$

and

$$
\max \left\{k: \gamma_{k}>0\right\}
$$

rather than the respective cardinality vectors; two sequences having the same value for

$$
\max _{i}\left|\delta_{i}\right|
$$

for example, are not necessarily of equal quality as judged by the first criterion, which also considers the number of integers in the sequence that exhibit this maximum absolute deviation.)

An alternative to selecting a sequence based on one of the two criteria that we have addressed is to randomly generate sequences of trials using, for example, the algorithm of Emerson and Tobias (1995), which samples from the vast number of feasible sequences with equal probability. Table 8 reports, for each $N=3, \ldots 9$, the quality (based on each criterion) of 1,000 sequences randomly generated by their codes. The histograms shown in Figures 1 and 2 for the case $N=5$ are typical of those for all values of $N$. Using either criterion, the expected quality of the random sequence is far worse than those presented in this paper.

\begin{tabular}{|c|c|c|c|c|c|c|c|c|c|}
\hline \multirow[b]{2}{*}{$N$} & \multicolumn{3}{|c|}{$\max _{i}\left|\delta_{i}\right|$} & \multicolumn{3}{|c|}{$\max \left\{k: \gamma_{k}>0\right\}$} & \multicolumn{3}{|c|}{$\max \left\{k: \gamma_{k}^{O}>0\right\}$} \\
\hline & $\mathrm{A}$ & B & $\mathrm{C}$ & A & B & $\mathrm{C}$ & A & B & $\mathrm{C}$ \\
\hline 3 & 2 & 4 & 2 & 2 & 2 & 2 & 2 & 2 & 2 \\
\hline 4 & 0 & 5 & 2 & 4 & 3 & 3 & 4 & 3 & 3 \\
\hline 5 & 0 & 20 & 7 & 9 & 4 & 4 & 9 & 4 & 4 \\
\hline 6 & 0 & 20 & 10 & 10 & 5 & 5 & 10 & 5 & 5 \\
\hline 7 & 1 & 44 & 18 & 13 & 6 & 6 & 13 & 6 & 6 \\
\hline 8 & 15 & 44 & 30 & 21 & 7 & 8 & 21 & 7 & 8 \\
\hline 9 & 22 & 50 & 45 & 32 & 8 & 11 & 32 & 8 & 11 \\
\hline
\end{tabular}

Table 7

Comparison of Sequences for Each Criterion

\begin{tabular}{|c|c|c|c|c|c|c|c|c|c|c|}
\hline \multirow[b]{2}{*}{$N$} & \multirow[b]{2}{*}{ Opt } & \multicolumn{4}{|c|}{$\max _{i}\left|\delta_{i}\right|$} & \multirow[b]{2}{*}{ Opt } & \multicolumn{4}{|c|}{$\max \left\{k: \gamma_{k}>0\right\}$} \\
\hline & & $\operatorname{Min}$ & $\overline{\operatorname{Max}}$ & $M$ & $S D$ & & $\overline{\mathrm{Min}}$ & Max & $M$ & $S D$ \\
\hline 3 & 2 & 2 & 8 & 4.81 & 1.96 & 2 & 2 & 3 & 2.36 & 0.48 \\
\hline 4 & 0 & 1 & 21 & 11.86 & 4.08 & 3 & 3 & 7 & 4.47 & 1.30 \\
\hline 5 & 0 & 3 & 43 & 23.31 & 7.26 & 4 & 4 & 13 & 7.43 & 2.06 \\
\hline 6 & 0 & 7 & 74 & 28.13 & 11.15 & 5 & 5 & 20 & 10.92 & 2.81 \\
\hline 7 & 1 & 16 & 111 & 59.90 & 16.73 & 6 & 7 & 27 & 14.69 & 3.64 \\
\hline 8 & 15 & 23 & 177 & 86.60 & 23.79 & 7 & 7 & 35 & 18.74 & 4.40 \\
\hline 9 & 22 & 47 & 242 & 123.10 & 32.04 & 8 & 12 & 44 & 23.76 & 5.65 \\
\hline
\end{tabular}

Table 8

Quality of Randomly Generated Sequences 


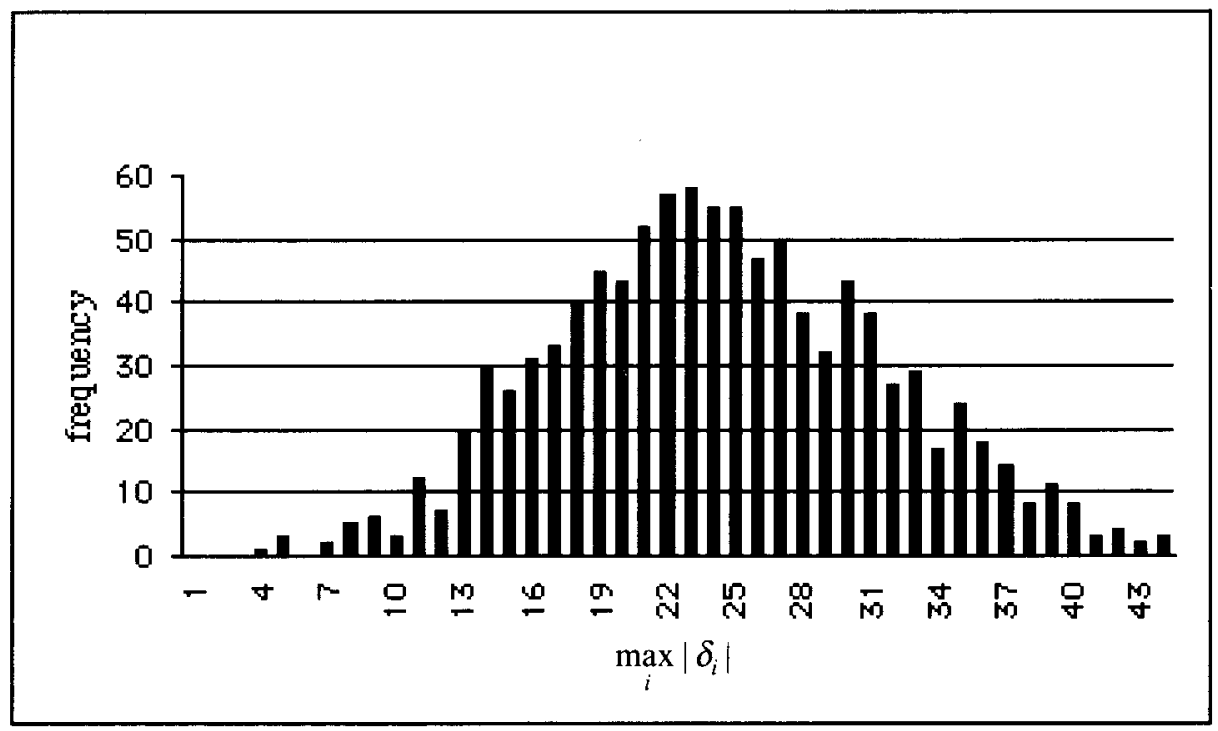

Figure 1. Frequencies of maximum absolute deviation $\delta$ from balance for 1,000 randomly generated sequences with $N=5$.

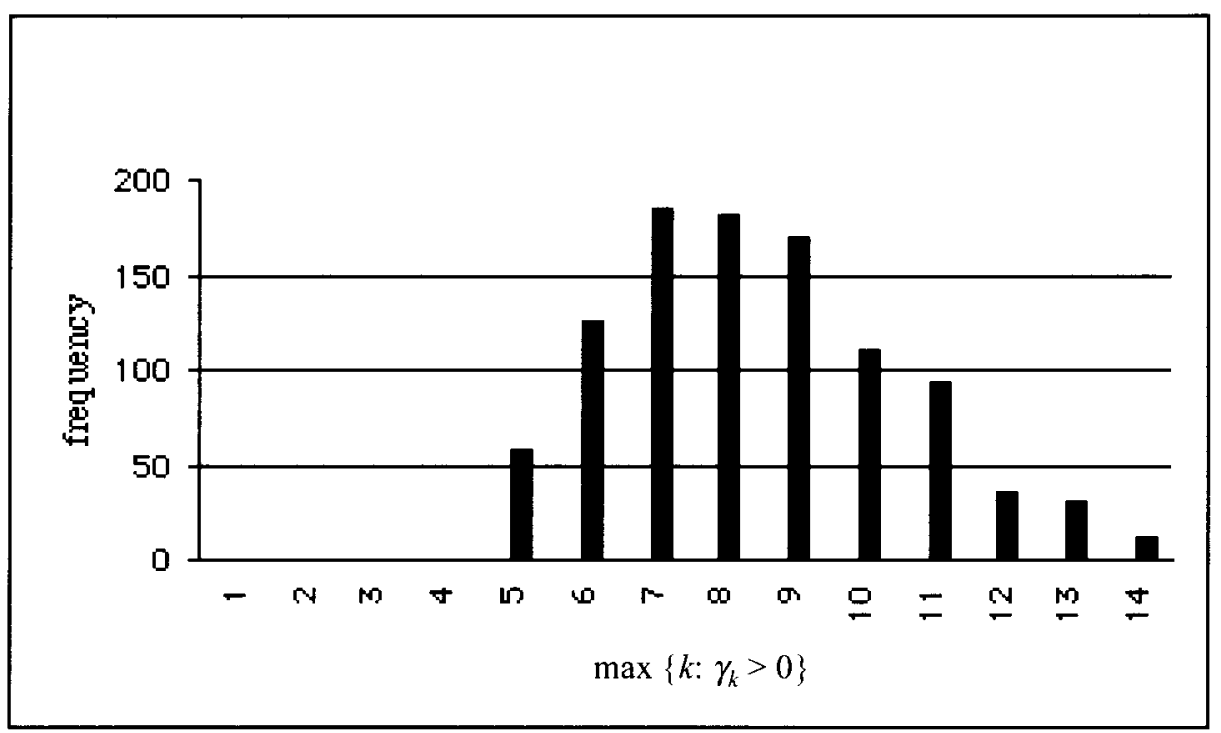

Figure 2. Frequencies of maximum deviation $\left|G_{i j}-N\right|$ of gaps for $\left|G_{i j}\right| 1,000$ randomly generated sequences with $N=5$.

Examination of Table 8 clearly indicates that for $N>3$, no randomly generated sequence exhibited a quality with respect to the balance criterion that was equal to the corresponding sequence in Table 1. A more detailed examination of the cardinality vectors of the random sequences for $N=3$ (for which only 216 sequences are feasible, so that each sequence is expected approximately five times) showed (i) that an optimal Type A sequence was generated 43 times, whereas an optimal Type B sequence was generated 137 times. For $N=4$, in which case 331,776 sequences are feasible, one optimal Type B sequence was found among the 1,000 randomly generated sequences.

\section{Availability}

A listing of the APL code used in generating the sequences reported in this report may be obtained by an e-mail request to the second author at dennis-bricker $(a)$ uiowa.edu. Additional sequences for $N>9$ are available via the World-Wide Web at http://www.icaen.uiowa.edu/ $\sim \mathrm{dbricker} /$ sequences.html

\section{REFERENCES}

Bertelson, P. (1961). Sequential redundancy and speed in a serial twochoice responding task. Quarterly Journal of Experimental Psuchologr $12,90-102$. 
Emerson, P. L., \& Tobias, R. D. (1995). Computer program for quasirandom stimulus sequences with equal transition frequencies. Behavior Research Methods, Instruments, \& Computers, 27, 88-98.

HinRICHS, J. V., \& CRAFT, J. L. (1971). Verbal expectancy and probability in two-choice reaction time. Journal of Experimental Psychol$\operatorname{og} y, 88,367-371$.

Pashler, H., \& Baylis, G. (1991a). Procedural learning: Intertrial repetition effect in speeded-choice tasks. Journal of Experimental Psychology: Learning, Memory, \& Cognition, 17, 33-48.

PASHleR, H., \& Baylis, G. (199l b). Procedural learning: Locus of practice effects in speeded choice-tasks. Journal of Experimental Psychologv: Learning. Memory, \& Cognition, 17, 20-32.
Sмiтн, M. C. (1968). Repetition effect and short-term memory. Journal of Experimental Psychology, 77, 435-439.

WiLliams, J. A. (1966). Sequential effects in disjunctive reaction time: Implications for decision models. Journal of Experimental Psychology, 71, 665-672.

WoODworth, R. S. (1938). Experimental psychology. New York: Holt.

(Manuscript received November 10, 1995; revision accepted for publication December 13, 1996. ) 